\title{
A novel particle source based on electrospray charging for dust accelerators and its significance for cosmic dust studies
}

\author{
Jonathan D. Kerby ${ }^{1 *}$, R. Terik Daly ${ }^{1,2 *}$, and Daniel E. Austin ${ }^{1}$ \\ ${ }^{1}$ Department of Chemistry and Biochemistry, Brigham Young University, Provo, Utah, U.S.A. \\ ${ }^{2}$ Department of Geological Sciences, Brigham Young University, Provo, Utah, U.S.A.
}

(Received November 7, 2011; Revised July 29, 2012; Accepted August 20, 2012; Online published March 12, 2013)

\begin{abstract}
In situ cosmic dust analyzers, like those aboard Cassini, Galileo, and Stardust, provide important data about cosmic dust, including the chemical composition of dust particles. Correctly interpreting the data from these cosmic dust analyzers requires laboratory calibration experiments. By studying the impact mass spectrum from a particle of known composition, an unknown particle's composition can be inferred from its impact mass spectrum measured in situ. This work expands a recently presented method, electrospray charging, introduced as a means of charging minerals, astrophysical ices, and mineral-ice mixtures that are not electrically conductive. This overcomes a limitation of current dust accelerators: they can only charge, and therefore accelerate, electrically conductive particles. This paper presents experiments demonstrating that microparticles (typically 2 micrometers) of olivine, a common mineral in meteorites, and of an ordinary chondrite (Allan Hills A 79045) can be electrically charged even though neither is electrically conductive. This article discusses the acceleration potential of electrospray-charged projectiles, and explores ways to overcome the challenges implicit in coupling an electrospray source to a dust accelerator. Electrospray charging may enable experiments with novel projectiles that are better analogues for cosmic dust than the conductive projectiles currently in use. Theoretical charging limits using this technique would allow micron-sized particles to be accelerated to several $\mathrm{km} / \mathrm{s}$ using 3 MV potential, with higher velocities for smaller particles.
\end{abstract}

Key words: Cosmic dust, dust accelerator, olivine, chondrite, microparticle impact, cosmic dust analyzer.

\section{Introduction}

Cosmic dust, including interstellar, interplanetary, and circumplanetary dusts, is a repository of information about Solar System history, processes, and bodies. Extracting and interpreting this information is a key goal of cosmic dust studies. Researchers use a variety of methods to study cosmic dust, ranging from studies of individual dust particles collected in the stratosphere or extracted from deep sea sediments (e.g., Brownlee, 1985) to detailed analyses of particles brought back by sample return missions (e.g., Brownlee et al., 2006) and large-scale studies using telescopes (e.g., Levasseurregourd and Dumont, 1980).

One technique for studying cosmic dust relies on in situ measurements by spacecraft. In situ cosmic dust analyzers have flown on a number of missions, including Galileo (Grün et al., 1992), Cassini (Srama et al., 2004), and Stardust (Kissel et al., 2004), and each of these analyzers has produced important results. For example, the cosmic dust analyzer on Cassini plays a key role in studies of Enceladus' plume and Saturn's E ring, providing information about particle production rate (Spahn et al., 2006) and plume compo-

*These students contributed equally to this work.

Copyright (C) The Society of Geomagnetism and Earth, Planetary and Space Sciences (SGEPSS); The Seismological Society of Japan; The Volcanological Society of Japan; The Geodetic Society of Japan; The Japanese Society for Planetary Sciences; TERRAPUB.

doi:10.5047/eps.2012.08.005 sition (Postberg et al., 2008). These data constrain the nature of Enceladus' interior (Postberg et al., 2009), which in turn informs discussions about the astrobiological potential of Enceladus. The Galileo Dust Detector provided critical information about Jupiter's rings, including the dust density in the Jovian system, the mass of the rings, and the relationships between Jovian moons and rings (Kruger et al., 2005, 2009). Finally, the Stardust mission's Cometary and Interplanetary Dust Analyzer (CIDA) revealed that organic compounds were a major component of the dust particles in the coma of Comet 81P/Wild 2 (Kissel et al., 2004).

In situ cosmic dust analyzers provide compositional information about dust particles and characterize particle masses, trajectories, and fluxes. The cosmic dust analyzers on Cassini, Galileo, and Stardust determined particle composition by the impact ionization of cosmic dust particles colliding with an impact plate in the dust analyzer. The chemical species produced by these hypervelocity impacts were extracted and analyzed using time-of-flight mass spectrometry (Grün et al., 1992; Kissel et al., 2004; Srama et al., 2004). Particle composition was determined by analyzing the mass spectra and inferring original particle composition from the chemical species observed upon impact ionization.

The mass spectra of the species produced by impact ionization are complex and difficult to interpret, which makes unraveling the dust particle's composition from mass spectra challenging. It is, however, an essential task critical to interpreting the data from these spacecraft. If the composi- 
tion of the impacting particle cannot be accurately deduced from the spectra of the impact ionized species, then it is impossible to obtain compositional information from in situ cosmic dust analyzers.

Calibration experiments in terrestrial labs lead to a better understanding of how to infer a particle's composition from its impact ionization mass spectrum. Calibration experiments often use dust accelerators to accelerate cosmic dust-sized projectiles to speeds comparable to the encounter velocity between the spacecraft instrument and dust particle. An accelerated microparticle collides with the impact plate of a flight spare or engineering equivalent of the spacecraft instrument, the chemical species created by impact are analyzed, and a mass spectrum is collected (e.g., Mocker et al., 2010). By studying the time-of-flight mass spectra of particles with a known composition, researchers determine how to interpret the mass spectra of particles with an unknown composition. These calibration experiments are a critical component of research programs supporting in situ cosmic dust analyzers, and it is advantageous to run calibration experiments with as many particle types as possible. Ideally, calibration experiments should use particles that are close analogues for actual cosmic dust particles. For example, since the Stardust CIDA encountered cometary ices, calibration experiments for this instrument would ideally be done with icy projectiles. The practical limitations of dust accelerators, however, prevent such experiments at present.

It is important to note that since cosmic dust analogues must be accelerated to hypervelocity speeds before being impacted and analyzed, the only materials that can be used as cosmic dust analogues are those that can be accelerated by dust accelerators. Electrostatic dust accelerators can only accelerate charged microparticles. Because existing dust accelerators charge projectiles through contact with a charged needle, only electrically conductive projectiles can be charged using this technique. This restriction limits the kinds of materials that can be charged, accelerated, and used to calibrate in situ cosmic dust analyzers. Most particles of interest, including stony meteorites, minerals, astrophysical ices, and mineral-ice mixtures, are not electrically conductive.

One approach to overcoming the conductive projectile requirement is to coat non-conductive particles with conductive materials, and Burchell et al. (1999), Goldsworthy et al. (2003), Srama et al. (2009), and Mocker et al. (2010) have succesfully used this method to increase the diversity of projectiles that can be charged by existing dust sources. However, this method cannot be applied to ices or mineralice mixtures. In addition, conductive coatings on nonconductive particles may disproportionately influence the mass spectra of ionized species produced by impact because of preferential surface ionization during microparticle impact, especially at lower velocities (Sysoev et al., 1992, 1997; Burchell et al., 1998; McDonnell et al., 2001). While conductive surface coatings do allow limited expansion of particle types, they also introduce artifacts into the particle's impact ionization mass spectrum-artifacts that may complicate applying results from calibration experiments to spacecraft data.

While significant progress has been made using conven- tional dust sources and dust accelerators, a dust source that overcomes the conductive limitation associated with contact charging could dramatically expand the types of materials usable as projectiles. This in turn would enable experiments with natural, uncoated solar system materials that are better analogues for actual cosmic dust than many of the conductive projectiles currently in use.

We recently reported (Daly et al., 2013) experiments demonstrating that quartz microparticles, methanol-water ices, and quartz-ice aggregates can be electrically charged using electrospray. Electrospray is a technique that can charge many types of particles, regardless of their electrical conductivity. Frequently used to charge large biomolecules for mass spectrometry, electrospray has also been applied to other particles, including inorganic compounds (Cole, 1997). This study expands on our previous work by applying electrospray charging to olivine and ordinary chondrite microparticles, samples arguably more relevant to cosmic dust than quartz. This article presents the results of these experiments, discusses the acceleration potential of electrospray-charged projectiles, and explores ways to overcome the challenges implicit in coupling an electrospray source to a dust accelerator.

\section{Methods}

The experimental setup involves three major components: an electrospray source to charge the microparticles (Fig. 1), a differential pumping system with heated, stainless steel beam tube, and an in vacuo image charge detector that quantifies the charge on electrosprayed microparticles. The experiments themselves use microparticles of various types suspended in a solvent. In this section we briefly discuss the experimental setup, which was described in detail by Daly et al. (2013), and the microparticles used in these experiments.

\subsection{Electrospray charging}

Electrospray is a versatile charging technique that is widely used in mass spectrometry (Cole, 1997). It has been applied to biological molecules and inorganic coordination complexes (Cole, 1997), and even intact viruses (Fuerstenau, 2003). However, prior to Daly et al. (2013), electrospray had not been applied to minerals. That study showed that quartz can be positively charged by electrospray, and this study shows that olivine and ordinary chondrite microparticles, along with olivine-ice and chondriteice mixtures, can also be positively charged by electrospray (see Section 3).

Electrospray involves three steps: creation of charged droplets containing microparticles, evaporation and bifurcation of droplets, and desolvation of the microparticles. For positive mode electrospray, an acidified solution is slowly pumped through a needle in a strong DC field of several kilovolts per centimeter, which causes the solution to break into tiny, charged droplets laden with excess protons. Solvent evaporates from the electrosprayed droplets as they move through the electric field toward a grounded plate, and eventually the droplets break apart when the charge on the droplet exceeds the Rayleigh limit, which is the maximum charge a liquid droplet can carry before breaking apart. Once the Rayleigh limit is exceeded, the droplet is unstable 


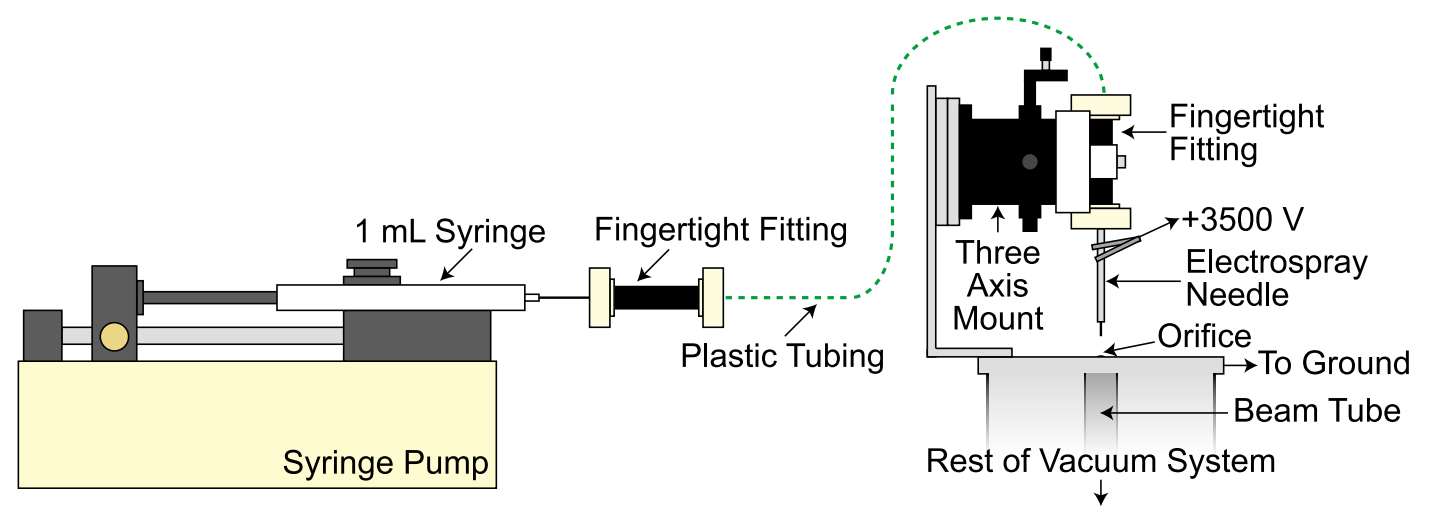

Fig. 1. Diagram showing a syringe pump, electrospray pump, and grounded orifice leading into vacuum (not to scale). The syringe pump dispenses the suspension to be electrosprayed at $300 \mu \mathrm{L} / \mathrm{hr}$. The syringe containing the suspension is connected to the electrospray needle by plastic tubing. The needle $(+3500 \mathrm{~V})$ is located $1.3 \mathrm{~cm}$ from a grounded orifice into vacuum. Electrosprayed droplets enter the vacuum system through a $150 \mu \mathrm{m}$ orifice where they pass through a $30 \mathrm{~cm}$ heated beam tube before entering the rest of the vacuum system.

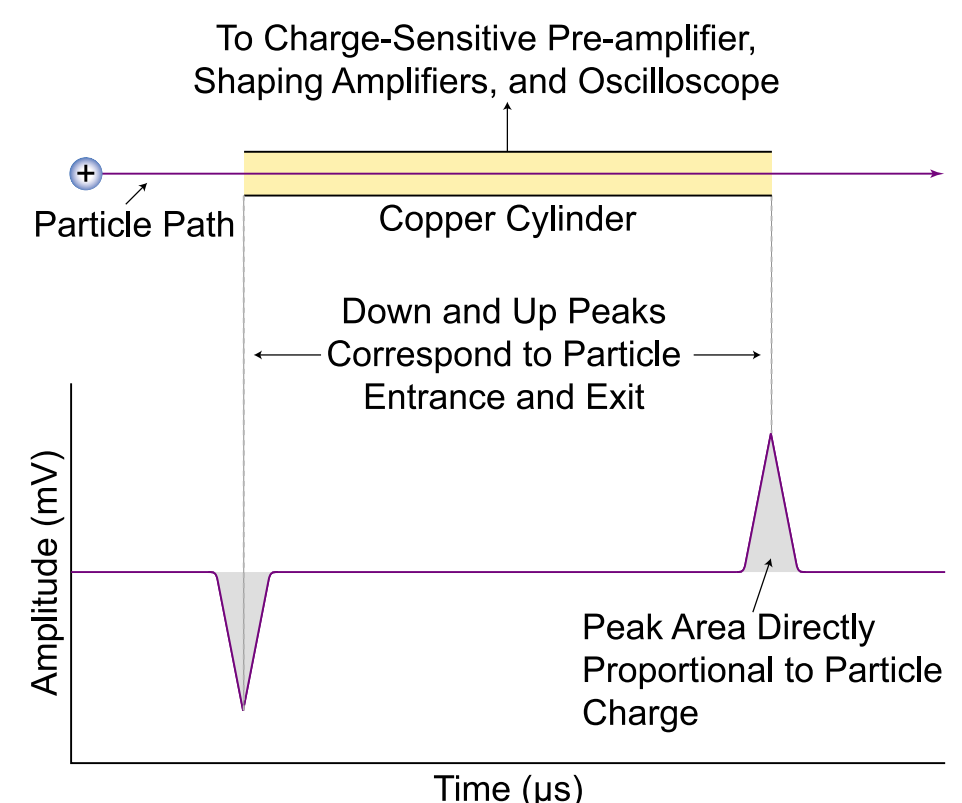

Fig. 2. The signal created by a charged particle passing through the detection cylinder of an image charge detector. For our circuit that uses a differentiating amplifier, peak area is directly proportional to particle charge, with the proportionality constant calculated by pulsing a known amount of voltage through the detector.

and fragments into a number of smaller droplets, each carrying some of the original droplet's mass and charge. Evaporation and bifurcation continues until the solute particle is completely desolvated, leaving behind a solvent-free, protonated particle. This process occurs over a wide range of particle sizes, from single molecules to microparticles, although some details of the mechanism may be different over this range (Cole, 1997).

\subsection{Vacuum system}

The syringe pump, syringe, and electrospray needle described in Section 2.1 are all located outside of vacuum. The vacuum system is a two-stage differential pumping system, detailed in Daly et al. (2013). Electrosprayed particles enter the instrument through a $150-\mu \mathrm{m}$ orifice, travel through a beam tube, pass through a skimmer, and are detected by an image charge detector. Whereas the previous experimental setup used an aluminum beam tube, this study uses a tube made of stainless steel.

\subsection{Image charge detection}

Image charge detection is used to verify that particles are being electrosprayed and to quantify the charge on electrosprayed particles. As a charged particle enters, passes through, and exits the image charge detector, it induces an image charge on a detection cylinder (Fig. 2). The detection cylinder is connected to charge-sensitive (Amptek A250) and pulse-shaping (Amptek A275) electronics, and the processed signal consists of a down peak as the particle enters the cylinder and an up peak as the particle leaves the cylinder. Peak area is directly proportional to particle charge (Maze et al., 2006; Mabbett et al., 2007; Zilch et al., 2008; Smith et al., 2011). Note that in some other charge detector instruments the charge is proportional to signal amplitudethe difference being the type of amplifier used. For our instrument, $1 \mu \mathrm{Vs}$ of peak area corresponds to 168,000 elementary charges. This peak area to particle charge conversion factor was calibrated by pulsing a known amount of 


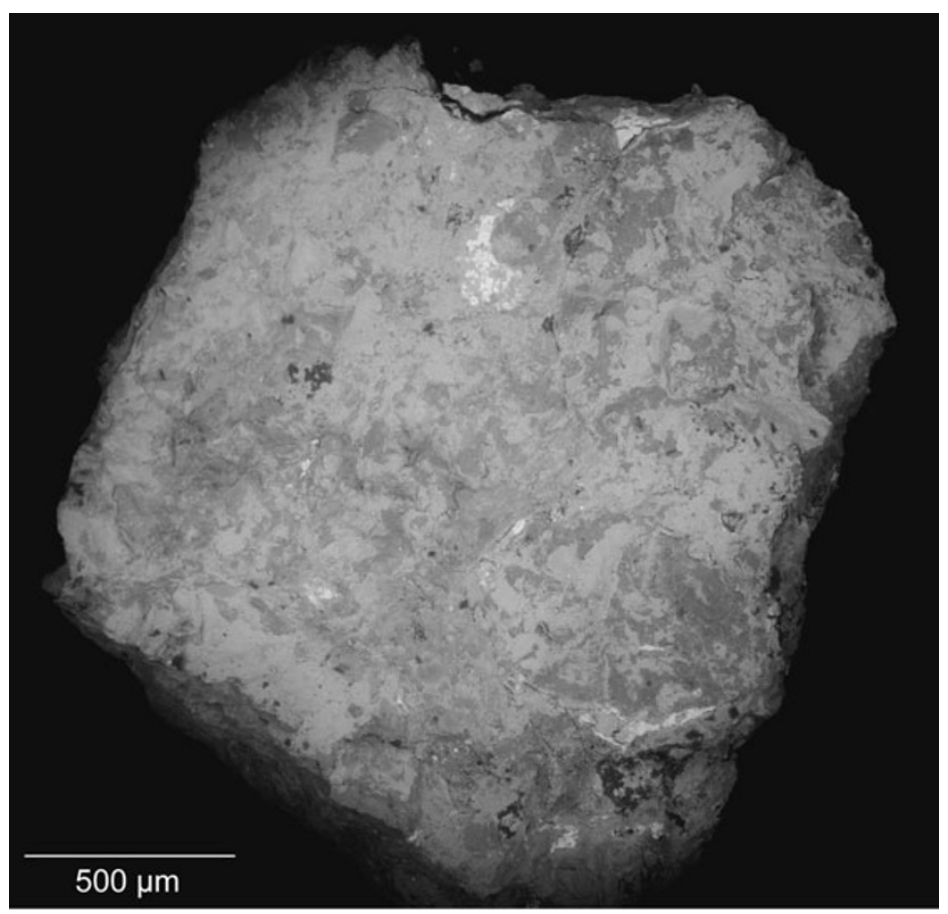

Fig. 3. Backscattered electron image of the allocated sample of ordinary chondrite ALHA 79045 . Sample mass was 9 mg. Energy dispersive spectroscopy revealed at least four phases in the sample. The brightest areas are rich in $\mathrm{Fe}$ and $\mathrm{S}$, the medium-bright areas are rich in Fe and $\mathrm{O}$, the medium-dark areas are rich in $\mathrm{Fe}, \mathrm{Mg}, \mathrm{Si}$, and $\mathrm{O}$, and the dark areas are $\mathrm{C}$-rich.

charge through the $2 \mathrm{pF}$ test circuit in the Amptek A250 and measuring the peak area of the processed signal. The calibration experiment also showed that the charge detector's performance is well behaved.

\subsection{Particle types}

This study focused on four particle types: olivine microparticles, ordinary chondrite microparticles, olivine-ice mixtures, and chondrite-ice mixtures. Olivine was used in experiments because it is a mineral frequently found in many stony meteorites and is thus a relevant analogue for such meteorites. However, most meteorites are multi-phase: they are rocks, and not minerals. Therefore, we also obtained a sample of a type L3.5 ordinary chondrite to use in experiments, as this is a better analogue for multi-phase stony meteorites than olivine alone.

2.4.1 Ordinary chondrite Allan Hills A 79045 The chondrite used in this study was Allan Hills A 79045, a chondrule-rich type L3 ordinary chondrite of weathering grade C (Grossman, 1994). The small ( $\sim 9 \mathrm{mg}$ ) sample obtained for this study was characterized by scanning electron microscopy and qualitative energy dispersive spectroscopy (EDS) before being crushed into $\sim 2 \mu \mathrm{m}$ diameter microparticles. The EDS work revealed that at least four phases are present (Fig. 3); however, since these EDS data are qualitative, it is not possible to specify the exact composition of these phases. The brightest phase in backscattered electron image is very rich in Fe and $\mathrm{S}$ and appears to rim chondrule fragments. The qualitative composition and spatial distribution of the phase are consistent with the troilite reported surrounding chondrules in ALHA 79045 (Score, 1981). A medium-bright phase is rich in $\mathrm{Fe}$ and $\mathrm{O}$; since $\mathrm{H}$ was not detected, this might be an iron oxide(s) or oxyhydroxide(s). "Limonitic staining" is reported in Score (1981). A medium-dark phase is rich in $\mathrm{Fe}, \mathrm{Mg}, \mathrm{Si}$, and $\mathrm{O}$ may be either olivine or pyroxene since these silicates are reported in Score (1981). The medium-bright and medium-dark phases appear to be the most common phases in the sample (Fig. 3). Lastly, the darkest phase is very C-rich, suggesting some sort of carbonaceous material.

2.4.2 Particle preparation Microparticles of olivine and ordinary chondrite were produced by first crushing the samples to $\sim 0.5 \mathrm{~mm}$ pieces and then grinding the pieces in a McCrone micronizing mill for 30 minutes. The micronizing mill is a wet slurry grinder that uses thirty agate grinding elements, and our protocol produces olivine and ordinary chondrite microparticles $\sim 2 \mu \mathrm{m}$ in diameter. After the samples were micronized, they were suspended in a 4 to 1 methanol-water solution to create a $0.005 \% \mathrm{w} / \mathrm{v}$ suspension of microparticles. This is not the only solvent combination that works for electrospray, but our experience, as well as the work of Cai et al. (2002), shows this solvent combination and particle concentration are effective. The suspensions were sonicated for thirty minutes to disperse the particles, and they were re-sonicated for at least two minutes before using them in an experiment. For the experiments in this study, the $\mathrm{pH}$ of both olivine and meteorite suspensions was 4.7 .

\section{Results}

Data indicate successful charging of both chondrite and olivine microparticles, along with chondrite-ice mixtures and olivine-ice mixtures. This section describes results from experiments done with pure solvent, olivine particles, chondrite particles, and olivine-ice and chondrite-ice aggregates. 


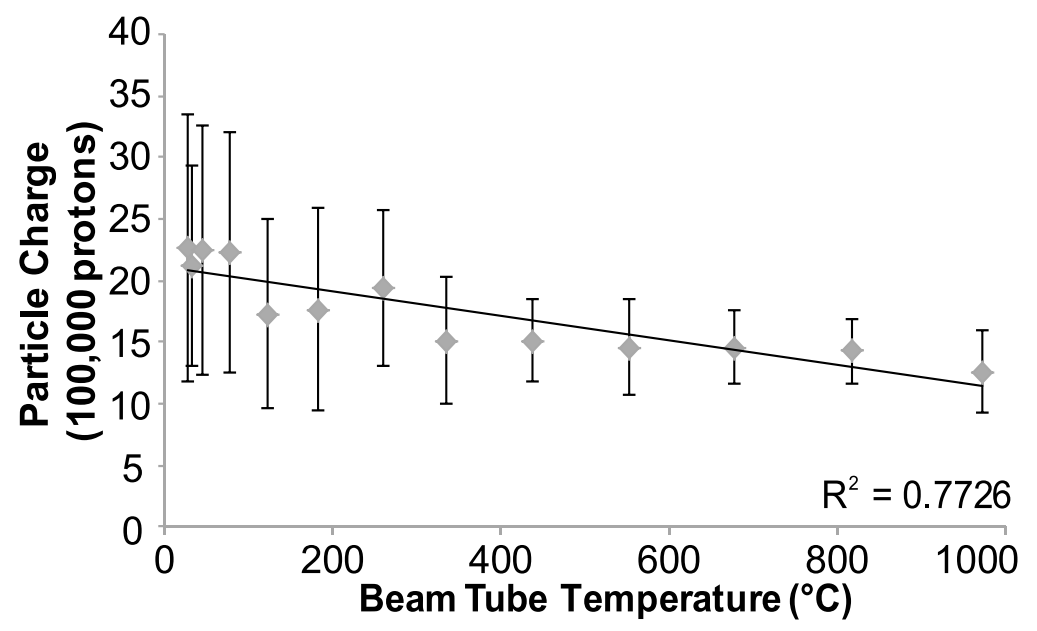

Fig. 4. Scatterplot showing the decrease in olivine particle charge with increasing beam tube temperature, indicating progressive desolvation of olivine microparticles. The error bars do not represent uncertainties in the measured value, but variation among the particles seen at those temperatures. Thus, at higher temperatures we do not see as many particles with as much charge, but we still see particles in the low charge range.

\subsection{Solvent-only particles}

Prior to experiments with olivine and chondrite microparticles, experiments were done using only the solvent in order to determine how beam tube temperature affected peak area, which provides information about particle desolvation as a function of beam tube temperature as described in Daly et al. (2013). Note that although the solvent particles are produced as liquid droplets at the electrospray source, the droplets freeze after the beam tube as they travel through vacuum. Thus, we infer that the charge detector is detecting frozen ice particles, not liquid droplets. We successfully charged and detected 555 solvent particles at beam tube temperatures of $26.2,30.6,44.1,75.3,120.3,181.9,260.0$, $333.2,437.8,551.0,677.8,818.4$, and $972.6^{\circ} \mathrm{C}$. Studying the relationship between beam tube temperature and particle charge enables a deeper understanding of how effective the beam tube is at desolvating particles.

The experiment done by Daly et al. (2013) indicate the charge of the particles decreased with increasing beam tube temperature, and in those experiments solvent particles were no longer detected at $609^{\circ} \mathrm{C}$; however, in this study particles were still detected above this temperature. This may be because the beam tube used in Daly et al. (2013) was aluminum and the beam tube used in these experiments was stainless steel, which has a lower thermal conductivity. The lower thermal conductivity of stainless steel may reduce heat transfer from the beam tube to the gas passing through the beam tube and, finally, to the electrospraycharged solvent ice particles. Alternatively, this might indicate a problem with the temperature calibration relating autotransformer output to beam tube temperature. Despite this difference, it is clear that peak area, and thus particle charge, decreases with increasing beam tube temperature.

\subsection{Olivine and chondrite particles}

Data from 690 olivine particles and 546 chondrite particles indicate successful charging of olivine particles, chondrite particles, olivine-ice mixtures, and chondrite-ice mixtures. Although it is not possible, with our instrument, to visually inspect these particles in vacuum, the very low concentration of particles in the electrospray suspension suggests that these particles are detected as individuals, not agglomerations (Cai et al., 2002). Desolvation experiments with the olivine particles show that particle charge decreases with increasing beam tube temperature, indicating progressive desolvation with increasing beam tube temperature (Fig. 4); however, the data also imply that a heated beam tube is not very efficient at desolvating these particles. This work confirms that a heated beam tube, while somewhat effective, is not an efficient desolvation mechanism. In addition to using a heated beam inside the vacuum system, a heated capillary outside the vacuum system or a sheath of nebulizing gas around the electrospray needle itself could also facilitate desolvation, and these methods should be more efficient than the heated beam tube.

We note that since our current concern is with charging the microparticles, we did not attempt electrical acceleration of the charged microparticles. The measured velocities of the particles are due only to aerodynamic effects of introducing them into vacuum.

The reason for the decrease in particle charge with increasing beam tube temperature can be partially explained by the ion evaporation model (Wang and Cole, 2000) for electrospray. The ion evaporation model holds that when electrosprayed droplets bifurcate, the bifurcated droplets are smaller in both size and charge relative to the primary droplets. Thus, as desolvation proceeds with the aid of the heated beam tube, the charges on the solvent-encased solute particles are divided among their progeny particles, which include solvent particles splitting off the solute particle. Each of the progeny particles has fewer protons than the primary droplets, and peak area (and particle charge) therefore decreases with progressive desolvation.

3.2.1 Chondrite findings Data collected on chondrite microparticles yielded some interesting results. Peak areas for these data fall into two different populations (Fig. 5). Ongoing work involves confirming this result and investigating its causes. Current hypotheses include (1) that these two populations correspond to two different phases in the meteorite or (2) that they are due to two different particle sizes. In support of the first hypothesis, we might 


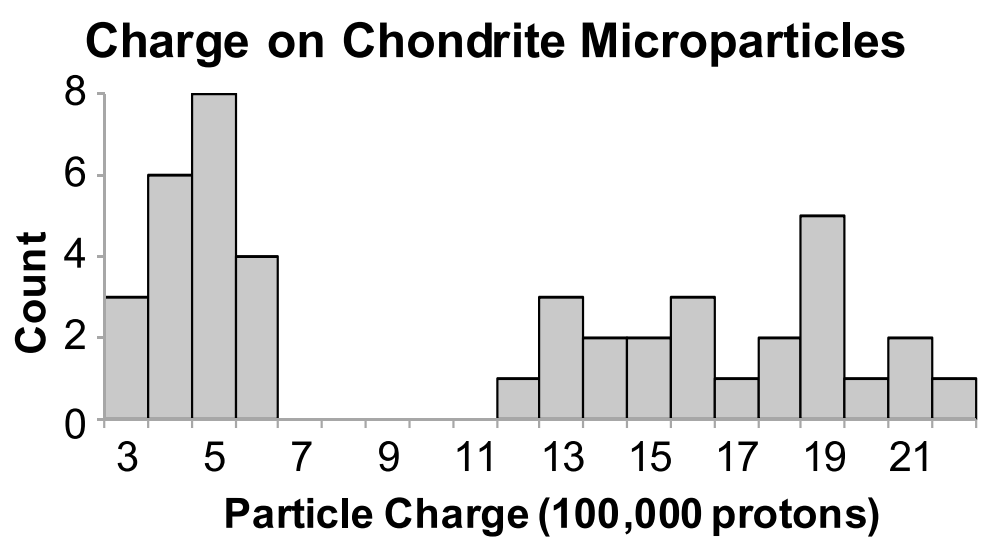

Fig. 5. Plot of the charged chondrite microparticles. Particle charges fall into two populations. The cause for these different populations is a topic of current investigation.

expect different phases in the meteorite to accept surface charge in different ways, leading to different final charges on the particles. However, if this hypothesis were correct, then we may expect four populations, instead of two, since the EDS data show four different phases in the meteorite sample. In support of the second hypothesis, we might expect that different phases in the meteorite would have different final particle sizes given the same grinding time due to the different crystallographic structures and hardness of the different phases. Further investigation is required to understand these observations.

\subsection{Olivine-ice and chondrite-ice aggregates}

When olivine and chondrite microparticles are electrosprayed without any heat on the beam tube, the microparticles are not completely desolvated, as indicated by the decrease in particle charge between an unheated beam tube and a beam tube heated to nearly $1000^{\circ} \mathrm{C}$ shown in Fig. 4. Instead, solvent evaporatively freezes onto the particle as it enters vacuum, creating an olivine-ice or chondrite-ice aggregate. Mineral-ice and rock-ice mixtures are quite relevant to cometary material and the particles of the Enceladus plume. In some ways, it is easier to create charged mineral-ice or rock-ice aggregates than to produce charged, completely desolvated mineral or rock microparticles. The issue is not that it is harder to charge these different types of particles; the issue is that completely removing the solvent from the solute particle can be challenging.

\section{Discussion}

\subsection{Implications}

These data, along with those in Daly et al. (2013), indicate that electrospray can charge a variety of planetary materials, including minerals, meteorites, mineral-ice mixtures, rock-ice mixtures, and ices. None of these particles are electrically conductive, but they were all successfully charged, indicating that electrospray charging overcomes the major limitation of contact charging. Since these particles can be charged by electrospray, they could be accelerated by dust accelerators, if an electrospray source were coupled to a dust accelerator.

We note that the successful charging of both quartz (Daly et al., 2013) and olivine in this study means that we have charged minerals from the two broad categories of min- eral surface charging behavior, as discussed in Kosmulski (2001). Since minerals from both of these surface charging categories have been charged by electrospray, it should be possible to use electrospray to charge most minerals. Likewise, successful electrospray charging of ordinary chondrites, which are aggregates of minerals, suggests that other multi-phase materials should also be charged. A third advantage of electrospray charging is that it can charge ices of variable composition. Although this study used a mixture of methanol and water for the solvent, other solvent combinations are possible, and using a new solvent is very easy. Thus, electrospray charging facilitates experiments with ices whose composition can be easily controlled and varied.

Electrospray charging suggests that hypervelocity microparticle impact experiments could be done using minerals, rocks, mineral-ice mixtures, rock-ice mixtures, and ices as projectiles, without the need for conductive surface coatings, if an electrospray source could be coupled to a dust accelerator. Such a coupling presents significant challenges, and we discuss some of those challenges in Subsection 4.4. Once such coupling takes place, however, researchers will be able to conduct impact ionization experiments with novel projectiles that are excellent analogues for cosmic dust without having to consider how conductive surface coatings affect experimental results. Such experiments may lead to better calibration of in situ cosmic dust analyzers and a clearer interpretation of the mass spectra from these instruments.

\subsection{Acceleration calculations}

Current dust accelerators use electric fields to accelerate charged microparticles to hypervelocity speeds, and a particle charged by electrospray should behave the same as a particle charged by contact, if it has the same mass to charge ratio. This section calculates the theoretical limit on the amount of charge that can be put on a particle by electrospray charging and determines the final velocities of particles accelerated through both $100 \mathrm{kV}$ and $3 \mathrm{MV}$ potential differences.

The amount of charge that can accumulate on an electrosprayed particle is limited by the Rayleigh limit-the maximum amount of charge on a droplet, beyond which the droplet fragments. The Rayleigh limit is a function of 


\section{Theoretical Final Velocity of Electrostatically Accelerated Electrospray-Charged Particles}

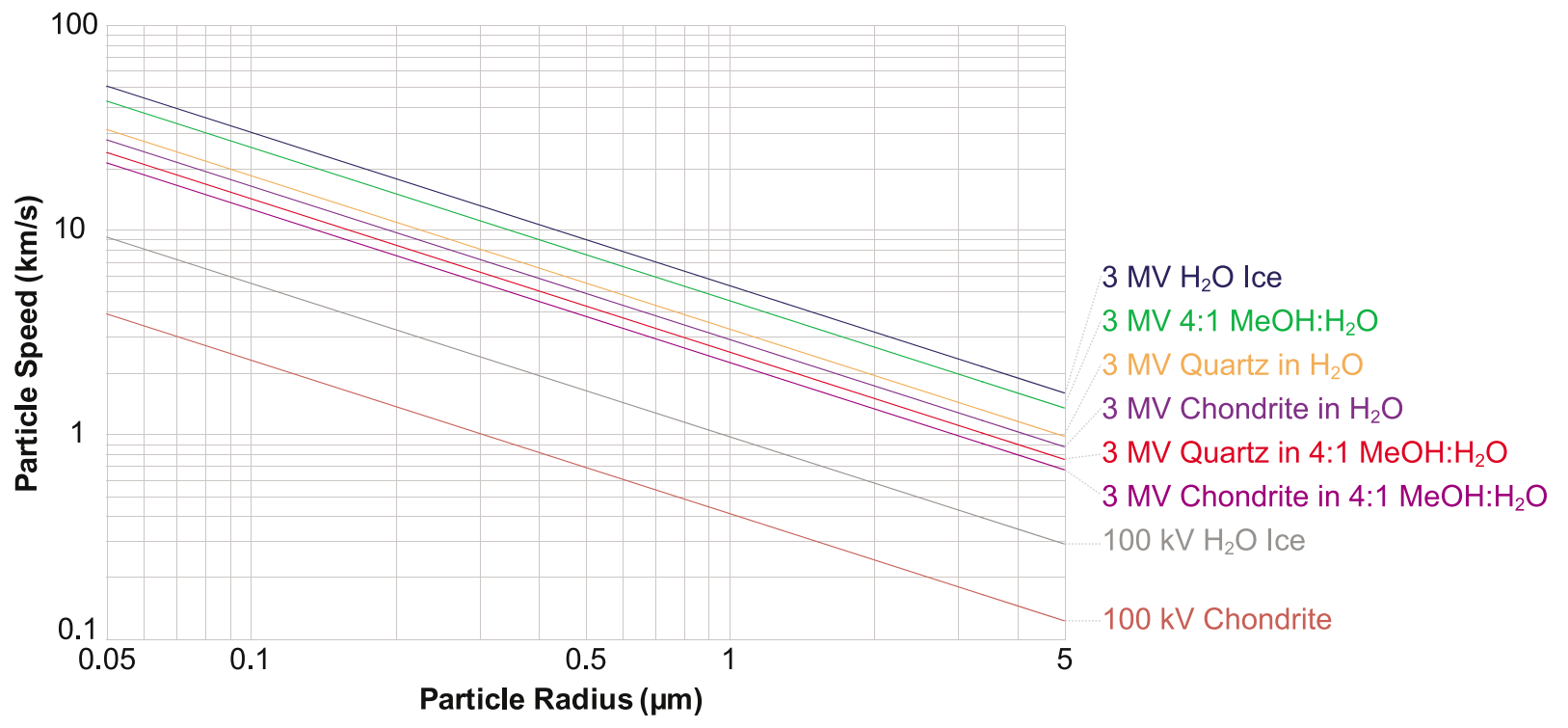

Fig. 6. Theoretical final velocities for various particle types, charged to the Rayleigh limit, accelerated through $100 \mathrm{kV}$ and $3 \mathrm{MV}$ potentials. Electrospray-charged particles can be accelerated to hypervelocity speeds, even with a modest $100 \mathrm{kV}$ accelertion potential. Note that the final velocities of olivine and chondrite microparticles are indistinguishable in this graph due to their similar densities.

Table 1. Inputs for acceleration calculations.

\begin{tabular}{cccc}
\hline Particle & Solvent & Surface tension $(\mathrm{mN} / \mathrm{m})$ & Density $\left(\mathrm{g} / \mathrm{cm}^{3}\right)$ \\
\hline $\mathrm{H}_{2} \mathrm{O}$ Ice & $\mathrm{H}_{2} \mathrm{O}$ & $71.99^{\mathrm{a}}$ & $0.9970470^{\mathrm{a}}$ \\
Chondrite & $\mathrm{H}_{2} \mathrm{O}$ & 71.99 & $3.35^{\mathrm{b}}$ \\
Olivine & $\mathrm{H}_{2} \mathrm{O}$ & 71.99 & $3.27^{\mathrm{c}}$ \\
Quartz & $\mathrm{H}_{2} \mathrm{O}$ & 71.99 & $2.65^{\mathrm{c}}$ \\
$4: 1 \mathrm{MeOH}: \mathrm{H}_{2} \mathrm{O}$ & $4: 1 \mathrm{MeOH}: \mathrm{H}_{2} \mathrm{O}$ & $25.54^{\mathrm{d}}$ & $0.8336^{\mathrm{d}}$ \\
Chondrite & $4: 1 \mathrm{MeOH}: \mathrm{H}_{2} \mathrm{O}$ & 25.54 & 3.35 \\
Olivine & $4: 1 \mathrm{MeOH}: \mathrm{H}_{2} \mathrm{O}$ & 25.54 & 3.27 \\
Quartz & $4: 1 \mathrm{MeOH}: \mathrm{H}_{2} \mathrm{O}$ & 25.54 & 2.65 \\
\hline${ }^{\mathrm{a}} \mathrm{Haynes}(2010)$. & & \\
${ }^{\mathrm{b}}$ Britt and Consolmagno (2003). & & \\
${ }^{\mathrm{c}}$ Klein and Dutrow (2008). & & \\
${ }^{\mathrm{d}} \mathrm{A}$ weighted average of values for methanol and water, respectively, from Haynes (2010).
\end{tabular}

droplet size and solvent surface tension.

The charge on a spherical water droplet charged to the Rayleigh limit, $q_{R_{y}}$ is

$$
q_{R_{y}}=8 \pi \sqrt{\varepsilon_{0} \gamma r^{3}} .
$$

Where $\varepsilon_{0}$ is the permittivity of vacuum, $\gamma$ is the surface tension of the solvent, and $r$ is the radius of the droplet (Cole, 1997). For an ice particle, which is simply frozen solvent, the surface tension in Eq. (1) is the surface tension of the solvent. The same is true for mineral-ice, rock-ice, mineral, and rock particles: the relevant surface tension in Eq. (1) is also the surface tension of the solvent. Thus, the Rayleigh limit of the solvent controls the maximum charge that can be applied to a particle.

The final velocities of microparticles, charged to the Rayleigh limit, accelerated through a $3 \mathrm{MV}$ potential are found by combining Eq. (1) with the equation for the velocity $v$ of a particle of mass $m$ and charge $q$ accelerated through a potential difference $V$ :

$$
v=\sqrt{\frac{2 V q}{m}} .
$$

To get a final equation:

$$
v=\sqrt{\frac{12 V \sqrt{\varepsilon_{0} \gamma r^{3}}}{\rho r^{3}}} .
$$

Thus, the final velocity $v$ of an electrosprayed particle accelerated through a potential $V$ is a function of the potential difference $V$, the solvent surface tension $\gamma$, the droplet (or particle) radius $r$, and particle density $\rho$.

We calculated final particle velocities for a range of particle types and sizes for both $100 \mathrm{kV}$ and $3 \mathrm{MV}$ acceleration potentials (Fig. 6 and Table 1). The $3 \mathrm{MV}$ potential is the maximum potential of state-of-the-art dust accelerators, such as the new instrument at the Colorado Center for Lunar Dust and Atmospheric Studies 
(lasp.colorado.edu/ccldas/facilities.html). With a $3 \mathrm{MV}$ potential, $200 \mathrm{~nm}$ particles can be accelerated to more than $10 \mathrm{~km} / \mathrm{s}$, and hypervelocity speeds are possible even with only $100 \mathrm{kV}$. Regardless of the electrostatic acceleration potential, final particle velocity decreases as particle size increases. We also note that increasing the surface tension of the solvent increases the charge on the particle and thus the particle's final velocity.

\subsection{Coupling an electrospray particle source to a dust accelerator}

The vast majority of laboratory simulations of cosmic dust impacts have relied on electrostatic dust accelerators, which unlike electrodynamic (synchronous) accelerators do not require knowing the mass-to-charge ratio of the particles. For microparticles, the mass-to-charge ratio varies from particle to particle, hence the utility of electrostatic acceleration. Higher velocities are achieved by using higher voltages, and most accelerators use 1-3 MV. However, preventing electrical discharges when working with such high voltages is challenging. The high-voltage ball is often surrounded by pressurized $\mathrm{CO}_{2}$ or $\mathrm{SF}_{6}$ that act to suppress discharges.

The challenge in using electrospray with an electrostatic accelerator stems from the need for differential pumping, i.e., the spray must be done at or near atmospheric pressure, and the gases pumped away. The source must reside within the high voltage ball, so gases must be exhausted or pumped through a large potential difference. At intermediate pressures (where the Paschen curve is at a minimum) discharge occurs at lower voltages.

One possible solution to this problem is to use a long pumping tube in which the electrical potential is gradually dropped using a series of electrodes connected by resistors. Differential pumping can be designed so that the different pressure regions avoid the minimum in the Paschen curve (Lieberman and Lichtenberg, 1994). Electrospray can be done directly into a $\mathrm{CO}_{2}$ atmosphere. Although even small amounts of solvent vapor reduce the breakdown voltage of $\mathrm{CO}_{2}$, it will still be higher than for air. Further, when water is used as the solvent, electrospray is more stable in $\mathrm{CO}_{2}$ than it is in air.

A more tenable solution to this problem is to electrospray directly into high vacuum from a heated capillary, as has recently been demonstrated for other applications (Ninomiya et al., 2011). An infrared laser can be used to desolvate particles in this case, or the particles can be left as mineral-ice mixtures.

Other challenges to doing electrospray within a highvoltage ball include sample access. Whereas conventional dust sources can run for weeks or longer with minimal need for access, electrospray systems will require more frequent service to replenish solvent, unclog spray tips, etc. Electrophoretic effects will be minimized as long as the entire source is contained within the high voltage region.

\section{Conclusions}

The results of this study and those of Daly et al. (2013) suggest that electrospray can charge a variety of minerals, rocks, mineral-ice mixtures, rock-ice mixtures, and astrophysical ices, and successful experiments have been done with minerals from both surface charging categories. Future work may include electrospray experiments with new minerals, rocks, or ices, but our current work focuses on coupling an electrospray source to a dust accelerator (see Subsection 4.3) and increasing the efficiency of particle desolvation.

Electrospray charging eliminates the conductive projectile limitation associated with contact charging dust sources, overcoming a significant limitation in current cosmic dust research. Based on theoretical calculations, electrospraycharged particles could be accelerated to velocities comparable to the encounter velocity between cosmic dust particles and in situ cosmic dust analyzers. If an electrospray source is coupled to a dust accelerator, it will be possible to do experiments with projectiles that are unprecedentedly good analogues for a wide range of cosmic dust particles. Such experiments may lead to better calibration of in situ cosmic dust analyzers, with a concomitant increase in our understanding of the data collected by these important spacecraft instruments.

Acknowledgments. Seth Call wrote software used to analyze charge detector data. Mike Standing of the Brigham Young University Microscopy Laboratory performed scanning electron microscope work. Derek Sears, Jani Radebaugh, and Eric Christiansen provided insightful suggestions. This work is funded by the Brigham Young University College of Physical and Mathematical Sciences.

\section{References}

Britt, D. T. and G. J Consolmagno, Stony meteorite porosities and densities: A review of the data through 2001, Meteorit. Planet. Sci., 38, 1161-1180, 2003

Brownlee, D. E., Cosmic dust-collection and research, Ann. Rev. Earth Planet. Sci., 13, 147-173, 1985.

Brownlee, D., P. Tsou, J. Aleon, C. M. O. Alexander, T. Araki, S. Bajt, G. A. Baratta, R. Bastien, P. Bland, P. Bleuet, J. Borg, J. P. Bradley, A. Brearley, F. Brenker, S. Brennan, J. C. Bridges, N. D. Browning, J. R. Brucato, E. Bullock, M. J. Burchell, H. Busemann, A. Butterworth, M. Chaussidon, A. Cheuvront, M. F. Chi, M. J. Cintala, B. C. Clark, S. J. Clemett, G. Cody, L. Colangeli, G. Cooper, P. Cordier, C. Daghlian, Z. R. Dai, L. D'Hendecourt, Z. Djouadi, G. Dominguez, T. Duxbury, J. P. Dworkin, D. S. Ebel, T. E. Economou, S. Fakra, S. A. J. Fairey, S. Fallon, G. Ferrini, T. Ferroir, H. Fleckenstein, C. Floss, G. Flynn, I. A. Franchi, M. Fries, Z. Gainsforth, J. P. Gallien, M. Genge, M. K. Gilles, P. Gillet, J. Gilmour, D. P. Glavin, M. Gounelle, M. M. Grady, G. A. Graham, P. G. Grant, S. F. Green, F. Grossemy, L. Grossman, J. N. Grossman, Y. Guan, K. Hagiya, R. Harvey, P. Heck, G. F. Herzog, P. Hoppe, F. Horz, J. Huth, I. D. Hutcheon, K. Ignatyev, H. Ishii, M. Ito, D. Jacob, C. Jacobsen, S. Jacobsen, S. Jones, D. Joswiak, A. Jurewicz, A. T. Kearsley, L. P. Keller, H. Khodja, A. L. D. Kilcoyne, J. Kissel, A. Krot, F. Langenhorst, A. Lanzirotti, L. Le, L. A. Leshin, J. Leitner, L. Lemelle, H. Leroux, M. C. Liu, K. Luening, I. Lyon, G. MacPherson, M. A. Marcus, K. Marhas, B. Marty, G. Matrajt, K. McKeegan, A. Meibom, V. Mennella, K. Messenger, S. Messenger, T. Mikouchi, S. Mostefaoui, T. Nakamura, T. Nakano, M. Newville, L. R. Nittler, I. Ohnishi, K. Ohsumi, K. Okudaira, D. A. Papanastassiou, R. Palma, M. E. Palumbo, R. O. Pepin, D. Perkins, M. Perronnet, P. Pianetta, W. Rao, F. J. M. Rietmeijer, F. Robert, D. Rost, A. Rotundi, R. Ryan, S. A. Sandford, C. S. Schwandt, T. H. See, D. Schlutter, J. Sheffield-Parker, A. Simionovici, S. Simon, I. Sitnitsky, C. J. Snead, M. K. Spencer, F. J. Stadermann, A. Steele, T. Stephan, R. Stroud, J. Susini, S. R. Sutton, Y. Suzuki, M. Taheri, S. Taylor, N. Teslich, K. Tomeoka, N. Tomioka, A. Toppani, J. M. Trigo-Rodríguez, D. Troadec, A. Tsuchiyama, A. J. Tuzzolino, T. Tyliszczak, K. Uesugi, M. Velbel, J. Vellenga, E. Vicenzi, L. Vincze, J. Warren, I. Weber, M. Weisberg, A. J. Westphal, S. Wirick, D. Wooden, B. Wopenka, P. Wozniakiewicz, I. Wright, H. Yabuta, H. Yano, E. D. Young, R. N. Zare, R. Zega, K. Ziegler, L. Zimmerman, E. Zinner, and M. Zolensky, Comet 81P/Wild 2 under a microscope, 
Science, 314, 1711-1716, 2006.

Burchell, M. J., M. J. Cole, and J. A. M. McDonnell, Role of particle charge in impact ionization by charged microparticles, Nuc. Inst. Mech. Phys. Res., 143, 311-318, 1998.

Burchell, M. J., M. J. Cole, S. F. Lascelles, M. A. Khan, C. Barthet, S. A. Wilson, D. B. Cairns, and S. P. Armes, Acceleration of conducting polymer-coated latex particles as projectiles in hypervelocity impact experiments, J. Phys. D., 32, 2758-2765, 1999.

Cai, Y., W. P. Peng, S. J. Kuo, Y. T. Lee, and H. C. Chang, Singleparticle mass spectrometry of polystyrene microspheres and diamond nanocrystals, Anal. Chem., 74, 232-238, 2002.

Cole, R B. (ed.), Electrospray Ionization Mass Spectrometry: Fundamentals, Instrumentation, and Applications, 577 pp., John Wiley and Sons, New York, 1997.

Daly, R. T., J. D. Kerby, and D. E. Austin, Electrospray charging of minerals and ices for hypervelocity impact research, Planet. Space Sci., 75, 182-187, 2013.

Fuerstenau, S. D., Whole virus mass analysis by electrospray ionization, $J$. Mass Spec. Soc. Jpn., 51, 50-53, 2003.

Goldsworthy, B. J., M. J. Burchell, M. J. Cole, S. P. Armes, M. A. Khan, S. F. Lascelles, S. F. Green, J. A. M. McDonnell, R. Srama, and S. W. Bigger, Time of flight mass spectra of ions in plasmas produced by hypervelocity impacts of organic and mineralogical microparticles on a cosmic dust analyser, Astron. Astrophys., 409, 1151-1167, 2003.

Grossman, J. N., The Meteoritical Bulletin, No. 76, January: The U.S. Antarctic Meteorite Collection, Meteoritics, 29, 100-143, 1994.

Grün, E., H. Fechtig, M. S. Hanner, J. Kissel, B. A. Lindblad, D. Linkert, D. Maas, G. E. Morfill, and H. A. Zook, The Galileo dust detector, Space Sci. Rev., 60, 317-340, 1992.

Haynes, W. M. (ed.), CRC Handbook of Chemistry and Physics, 91st ed., 2610 pp., CRC Press, Florida, 2010.

Kissel, J., F. R. Krueger, J. Silen, and B. C. Clark, The cometary and interstellar dust analyzer at comet 81P/Wild 2, Science, 304, 17741776, 2004.

Klein, C. and B. Dutrow, Manual of Mineral Science, 23rd ed., 675 pp., John Wiley and Sons, New York, 2008.

Kosmulski, M., Chemical Properties of Material Surfaces, 776 pp., CRC Press, New York, 2001.

Kruger, H., G. Linkert, D. Linkert, R. Moissl, and E. Grun, Galileo longterm dust monitoring in the jovian magnetosphere, Planet. Space Sci., 53, 1109-1120, 2005.

Kruger, H., D. P. Hamilton, R. Moissl, and E. Grun, Galileo in-situ dust measurements in Jupiter's gossamer rings, Icarus, 203, 198-213, 2009.

Levasseurregourd, A. C. and R. Dumont, Absolute photometry of zodiacal light, Astron. Astrophys., 84, 277-279, 1980.

Lieberman, M. A. and A. J. Lichtenberg, Principles of Plasma Discharges and Materials Processing, 600 pp., John Wiley and Sons, New York, 1994.

Mabbett, S. R., L. W. Zilch, J. T. Maze, J. W. Smith, and M. F. Jarrold, Pulsed acceleration charge detection mass spectrometry: Application to weighing electrosprayed droplets, Anal. Chem., 79, 8431-8439, 2007.

Maze, J. T., T. C. Jones, and M. F. Jarrold, Negative droplets from positive electrospray, J. Phys. Chem. A, 110, 12607-12612, 2006.

McDonnell, J. A. M., E. D. Aguti, and M. J. Willis, Pre-contact microdischarge from charged particulates, in Spacecraft Charging TechnologyProceedings of the Seventh International Conference, edited by R. A. Harris, 657 pp., ESA SP-476, 2001.

Mocker, A., S. Armes, S. Bugiel, K. Fiege, E. Gruen, B. Heines, J. Hillier,
S. Kempf, and R. Srama, The Heidelberg dust accelerator: Investigating hypervelocity particle impacts, Proceedings of the 2010 American Geophysical Union Fall Meeting, San Francisco, California, abstract no. P31B-1524, 2010.

Ninomiya, S., L. C. Chen, Y. Sakai, and K. Hiraoka, Vacuum electrospray assisted by laser irradiation, The 59th Annual Conference of the American Society for Mass Spectrometry, 5-9 June 2011, Denver, Colorado, abstract no. 1061, 2011.

Postberg, F., S. Kemp, J. K. Hillier, R. Srama, S. F. Green, N. McBride, and E. Grun, The E-ring in the vicinity of Enceladus II. Probing the moon's interior-The composition of E-ring particles, Icarus, 193, 438-454, 2008.

Postberg, F., S. Kempf, J. Schmidt, N. Brilliantov, A. Beinsen, B. Abel, U. Buck, and R. Srama, Sodium salts in E-ring ice grains from an ocean below the surface of Enceladus, Nature, 459, 1098-1101, 2009.

Score, R., C. M. Schwarz, T. V. V. King, B. Mason, D. D. Bogard, and E. M. Gabel, Antarctic Meteorite Newsletter, 4(1), 1981.

Smith, J. W., E. E. Siegel, J. T. Maze, and M. F. Jarrold, Image charge detection mass spectrometry: Pushing the envelope with sensitivity and accuracy, Anal. Chem., 83, 950-956, 2011.

Spahn, F., J. Schmidt, N. Albers, M. Horning, M. Makuch, M. Seiss, S. Kempf, R. Srama, V. Dikarev, S. Helfert, G. Moragas-Klostermeyer, A. V. Krivov, M. Sremcevic, A. J. Tuzzolino, T. Economou, and E. Grun, Cassini dust measurements at Enceladus and implications for the origin of the E ring, Science, 311, 1416-1418, 2006.

Srama, R., T. J. Ahrens, N. Altobelli, S. Auer, J. G. Bradley, M. Burton, V. V. Dikarev, T. Economou, H. Fechtig, M. Gorlich, M. Grande, A. Graps, E. Grun, O. Havnes, S. Helfert, M. Horanyi, E. Igenbergs, E. K. Jessberger, T. V. Johnson, S. Kempf, A. V. Krivov, H. Kruger, A. Mocker-Ahlreep, G. Moragas-Klostermeyer, P. Lamy, M. Landgraf, D. Linkert, G. Linkert, F. Lura, J. A. M. McDonnell, D. Mohlmann, G. E. Morfill, M. Muller, M. Roy, G. Schafer, G. Schlotzhauer, G. H. Schwehm, F. Spahn, M. Stubig, J. Svestka, V. Tschernjawski, A. J. Tuzzolino, R. Wasch, and H. A. Zook, The Cassini cosmic cust analyzer, Space Sci. Rev., 114, 465-518, 2004.

Srama, R., F. Postberg, M. Trieloff, J. K. Hillier, Z. Gainsforth, A. J. Westphal, S. Bugiel, E. Grün, S. Armes, A. Kearsley, T. Tyliszczak, and W. H. Schwarz, High velocity Van-de-Graff shots with mineral dust: An application for Stardust and other in-situ space missions, Proceedings of the 2009 AAS/Division for Planetary Sciences Meeting, Puerto Rico, abstract no. 41, 2009.

Sysoev, A. A., D. R. Bandura, and V. P. Ivanov, Mechanism of ionization in a low-velocity collision of charged microparticles, Sov. Tech. Phys. Lett., 18, 486-488, 1992.

Sysoev, A. A., V. P. Ivanov, T. V. Barinova, Y. A. Surkov, and V. V. Vysochkin, Mass spectra formation from charged microparticles, Nuc. Inst. Meth. Phys. Res. B, 122, 79-83, 1997.

Wang, G. and R. B. Cole, Charged residue versus ion evaporation for formation of alkali metal halide cluster ions in ESI, Anal. Chim. Acta, 406, 53-65, 2000.

Zilch, L. W., J. T. Maze, J. W. Smith, G. E. Ewing, and M. F. Jarrold, Charge separation in the aerodynamic breakup of micrometer-sized water droplets, J. Phys. Chem. A, 112, 13352-13363, 2008.

J. D. Kerby, R. T. Daly, and D. E. Austin (e-mail: austin@chem.byu.edu) 